\title{
Topological Transition in $\mathrm{Pb}_{1-x} \mathrm{Sn}_{x} \mathrm{Se}$ Using Meta-GGA Exchange-Correlation Functional Acta Physica Polonica A 136, 667 (2019), ERRATUM
}

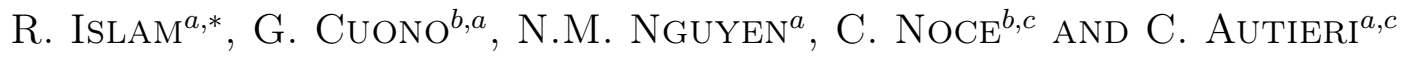 \\ ${ }^{a}$ International Research Centre Magtop, Institute of Physics, Polish Academy of Sciences, \\ Aleja Lotników 32/46, PL-02668 Warsaw, Poland \\ ${ }^{b}$ Dipartimento di Fisica "E.R. Caianiello", Università degli Studi di Salerno, \\ I-84084 Fisciano (SA), Italy \\ ${ }^{c}$ Consiglio Nazionale delle Ricerche CNR-SPIN, UOS Salerno, I-84084 Fisciano (Salerno), Italy \\ orginal Doi: 10.12693/APhysPolA.136.667*e-mail: rajibul.islam@magtop. ifpan.edu.pl \\ actual Doi: 10.12693/APhysPolA.139.169
}

\begin{abstract}
We calculate the mirror Chern number (MCN) and the band gap for the $\mathrm{Pb}_{1-x} \mathrm{Sn}_{x}$ Se alloy as a function of the concentration $x$ by using a virtual crystalline approximation. We use the electronic structure from the relativistic density functional theory calculations in the Generalized-Gradient-Approximation (GGA) and meta-GGA approximation. Our results obtained with the modified Becke-Johnson metaGGA functional are comparable with the available experimental data for the MCN as well as for the band gap. We advise to use the modified Becke-Johnson approximation with the parameter $c=1.10$ to describe the transition from a trivial to topological phase for this class of compounds.
\end{abstract}

topics: $31.10 .+\mathrm{z}, 73.43 . \mathrm{Nq}, 31.15 . \mathrm{E}-$

This article was originally published in October 2019 with erroneously swapped captions of Figs. 1-5. The captions of Figs. 1-5 should be corrected in the following way:

- Fig. 1 should have the caption of Fig. 5,

- Fig. 2 should have the caption of Fig. 1 ,

- Fig. 3 should have the caption of Fig. 2,

- Fig. 4 should have the caption of Fig. 3,

- Fig. 5 should have the caption of Fig. 4.

The Editors apologize for this error. 